\title{
Influence of Janus Kinase Inhibitors on the Neuronal Activity as a Proof-of-Concept Model for Itch
}

\author{
Johannes Wohlrab ${ }^{a, b}$ David Stintzing ${ }^{b}$ Luise Schultz ${ }^{c}$ Konstantin Jügelt ${ }^{c}$ \\ Olaf H.-U. Schroeder ${ }^{\mathrm{C}}$ \\ aDepartment of Dermatology and Venereology, Martin Luther University Halle-Wittenberg, Halle (Saale), Germany; \\ ' Institute of Applied Dermatopharmacy, Martin-Luther-University Halle-Wittenberg, Halle (Saale), Germany; \\ 'NeuroProof GmbH, Rostock, Germany
}

\section{Keywords}

Janus kinase · Janus kinase inhibitors · Itch · Neuronal activity

\begin{abstract}
Background: Itching is considered to be a subjective symptom of the activation of neurosensory structures by different signal molecules and trigger factors. The signaling cascades responsible for it are closely linked to inflammatory processes. This explains why itching also occurs in many inflammatory diseases. One of these signaling cascades is mediated by Janus kinases (JAKs). Recently, it could be shown on a molecular level that Janus kinase 1 (JAK1) directly activates frontal cortex neurons and thus can cause chronic itching. Objectives: This study deals with the influence of different JAK inhibitors (JAKi) on the activity of chip-based neural networks of cultured frontal cortex neurons by investigating neurophysiological activity parameters. This in vitro model provides information on dose-dependent effects of model substances with different specificity regarding the inhibition of different JAKs. Methods: Tofacitinib (pan-JAKi), baricitinib (JAK1/2i), and upadacitinib (JAK1i) in a concentration range from $10 \mathrm{nmol} / \mathrm{L}$ to $50 \mu \mathrm{mol} / \mathrm{L}$ were tested in a microelectrode
\end{abstract}

karger@karger.com www.karger.com/spp

Karger $\stackrel{\text { ' }}{5}$

GOPEN ACCESS
(C) 2021 The Author(s)

Published by S. Karger AG, Basel

This article is licensed under the Creative Commons Attribution 4.0 International License (CC BY) (http://www.karger.com/Services/ OpenAccessLicense). Usage, derivative works and distribution are permitted provided that proper credit is given to the author and the original publisher. array neurochip culture system. Results: The results show that the inhibition of the neuronal activity of frontal cortex neurons increases with JAK1 selectivity and is dependent on concentration. Conclusion: These observations are supported by data from clinical studies in atopic dermatitis and psoriasis. The clinical relevance of these results must be proven by further clinical studies with subjective and objective parameters for itching.

(c) 2021 The Author(s)

Published by S. Karger AG, Basel

\section{Introduction}

Chronic itching is a subjectively felt symptom, an independent sensory perception, which is based on a complex etiology of a disturbed neuronal activity [1]. The neurosensory signal is transmitted from the periphery via the spinal cord to the brain by primary afferent fibers (pruriceptors) [2]. These pseudounipolar and polymodal pruriceptors are divided according to biophysical criteria into thinly myelinated A (diameter $2-5 \mu \mathrm{m}$, conduction velocity up to $8 \mathrm{~m} / \mathrm{s}$ ) and unmyelinated C fibers (diameter $0.2-1.5 \mu \mathrm{m}$, conduction velocity $<2 \mathrm{~m} / \mathrm{s}$ ) [3]. They are integrated into an interactive environment that is regulated 


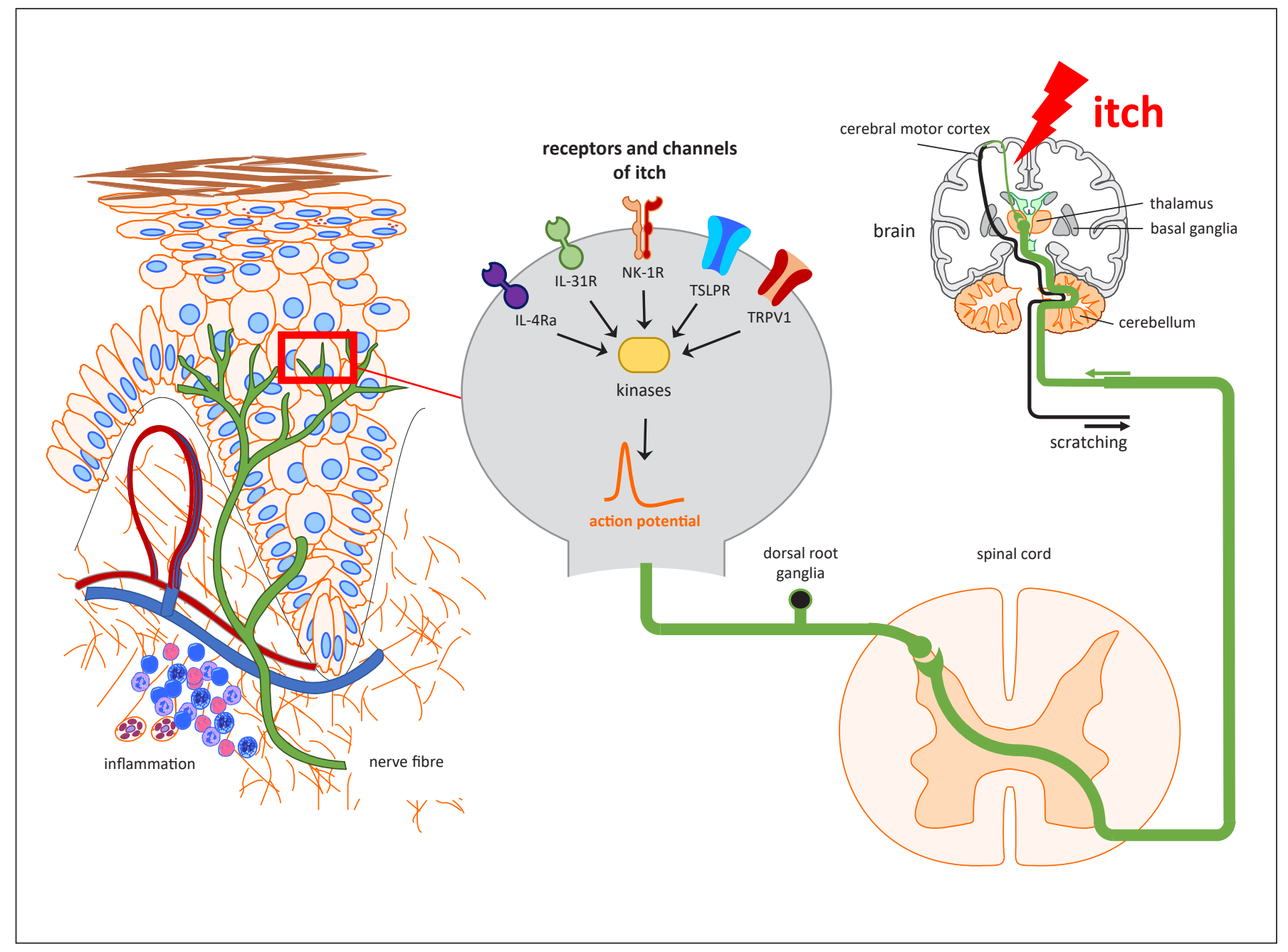

Fig. 1. Overview of the different receptor types of primary afferent sensory neurons, as well as cascades, during itching. IL-4R, interleukin-4 receptor subunit a; IL-31R, interleukin-31 receptor; NK-1R, neurokinine-1 receptor; TSLPR, thymic stromal lymphopoietin receptor; TRPV1, transient receptor potential cation channel subfamily $\mathrm{V}$ member 1 .

and determined by molecular (e.g., cytokines and ion channels), chemical (e.g., pH value), physical (e.g., temperature and tissue tension), and cellular (e.g., lymphocytes and macrophages) environmental conditions [4]. This makes it clear that inflammatory processes in the skin and their milieu can have a direct influence on the functional state of the pruriceptors.

This direct interaction has recently been proven at the molecular level for the JAK/STAT pathway too [5]. It is a cellular pathway of ligand-induced signal transduction activating the transmembrane receptor-associated Janus kinases, which via phosphorylation of the intracellular portions make different signal transducers and activators of transcription (STATs) available. These penetrate the cell nucleus and regulate gene expression by binding directly to the DNA. Various Janus kinases (JAK1-3 and TYK2) and their selective binding to respective receptor chains activate different STATs (STAT1-6) and thus induce special gene expression patterns [6]. This direct, simple, and yet variable signal transduction pathway is evolutionary very old and is equally important for basal regulation processes of the cell as well as for inflammation and neuroimmunological processes.

For neurotransmitter-mediated signaling, the integration of the JAK/STAT pathways has been described for both the central part (glial cells and astrocytes) $[7,8]$ and the peripheral part (DRGs) [9] of the neurosensory signal cascade. To date, the functional significance of the indi- 
vidual JAK subtypes is largely unknown. However, it is known that the JAK/STAT pathway, in particular JAK2/ STAT3, is also involved in neuroregenerative processes [10]. At the same time, the functional interconnection of JAK/STAT activation with immunological or inflammatory reaction patterns has been recognized and is currently being intensively studied and validated on molecular and immunological levels $[5,11]$. Regarding dermatological diseases, the focus of interest lies in the complex pathophysiology of itching and the functional integration of the JAK/STAT signaling pathway $[3,12,13]$. The JAK/ STAT signaling pathway is involved in stimulus processing both in the periphery as well as on the spinal cord and in the brain $[14,15]$. The blockage of the afferences is possible on different levels (Fig. 1). This applies in particular to JAKi, which pass the blood-brain barrier proportionally and also induce central effects [16]. However, the passage mechanism is largely unclear, even if p-glycoprotein has been identified as a possible transport protein [17, 18]. Although clinical data are available on the efficient reduction of itching by different JAKi, in particular for atopic dermatitis [19], psoriasis [20], and myelofibrosis $[21,22]$, clear evidence for the functional effects of JAKi in neurophysiological models is missing so far. Because the selectivity of the individual JAKi differs with respect to the inhibitory activity of the JAK subtypes, compounds with different inhibitory patterns have been selected as examples for the present proof-of-concept studies of the effects (IC50 values [JAK1, JAK2, JAK3, TYK2 - tofacitinib: $15 \mathrm{nmol} / \mathrm{L}, 77 \mathrm{nmol} / \mathrm{L}, 55 \mathrm{nmol} / \mathrm{L}$, and $489 \mathrm{nmol} / \mathrm{L}$; baricitinib: $4 \mathrm{nmol} / \mathrm{L}, 7 \mathrm{nmol} / \mathrm{L}, 787 \mathrm{nmol} / \mathrm{L}$, and 61 $\mathrm{nmol} / \mathrm{L}$; upadacitinib: $8 \mathrm{nmol} / \mathrm{L}, 600 \mathrm{nmol} / \mathrm{L}, 139 \mathrm{nmol} / \mathrm{L}$, and $4.700 \mathrm{nmol} / \mathrm{L}])$ [23].

\section{Materials and Methods}

\section{Test Substances}

JAKi with different specificity were selected as model substances. Tofacitinib citrate (Sigma-Aldrich, Darmstadt, Germany) as pan-JAKi, baricitinib (Sigma-Aldrich) as JAK1/2i, and upadacitinib (Sigma-Aldrich) as JAK1i dissolved in dimethyl sulfoxide (DMSO) (Sigma-Aldrich) at concentrations of 1, 10, 30, and 50 $\mu \mathrm{mol} / \mathrm{L}$ were examined. Concentrations were cumulatively increased. DMSO was included as a negative control $(0.002,0.02$, 0.06 , and $0.1 \%)$.

\section{Primary Cell Culture}

Experiments were carried out in accordance with the EU Directive 2010/63/EU on the protection of animals used for scientific purposes (Certification File No. 7221.3-2). The frontal cortex was harvested from embryonic day 16 chr:NMRI mice (Charles River,
Sulzfeld, Germany). Mice were sacrificed by cervical dislocation according to the German Animal Protection Act $\$ 4$. Tissue was dissociated by enzymatic digestion (133.3 Kunitz units/mL DNase; 10 units/mL papain) and mechanical trituration, counted, vitality controlled, and suspended in Dulbecco's modified essential medium containing laminin $(10 \mu \mathrm{g} / \mathrm{mL}), 10 \%$ fetal bovine serum as a seeding supplement, and $10 \%$ horse serum. Drops of $20 \mu \mathrm{L}$ were plated on multiwell microelectrode arrays (MEAs) [24]. The 48well MEA neurochips (Axion Biosystems, Atlanta, GA, USA) have 16 passive electrodes per well. The surface was coated with polyethyleneimine (50\% in borate buffer) for $1 \mathrm{~h}$, washed, and air-dried. Cultures on MEAs were incubated at $37^{\circ} \mathrm{C}$ in a $10 \% \mathrm{CO}_{2}$ atmosphere until ready for use. Culture media were replenished 2 times a week with Dulbecco's modified essential medium containing only $10 \%$ horse serum. The developing co-cultures were treated with the mitosis inhibitor 5-fluoro-2'-deoxyuridine (25 $\mu \mathrm{M})$ and uridine (63 $\mu \mathrm{M})$ on day 5 after seeding to prevent further glial proliferation.

\section{Multichannel Recording}

We used the multichannel recording system MAESTRO (Axion Biosystems) that provides sampling at $12.5 \mathrm{kHz}$, filtering, spike detection, and recording of extracellular spikes. MEAs were kept at $37^{\circ} \mathrm{C}$. The $\mathrm{pH}$ was maintained at 7.4 with a continuous stream of filtered, humidified airflow with $10 \% \mathrm{CO}_{2}$. We tested all compounds acutely on cultures after 28 days in vitro in the culture medium without medium exchanges. After recording the baseline activity for $120 \mathrm{~min}$ (which serves as an internal activity reference control for each experiment), the solvent control or test compounds were added at cumulatively increased concentrations. Compound stock solutions were pipetted into a 48-well mirror plate. Hundred microliters of medium was aspirated from wells with a multichannel pipette, mixed with the compounds, and returned to the respective well. The process did not exceed 3-5 min. The response was followed for $60 \mathrm{~min}$ after every application, of which the last $30 \mathrm{~min}$ was used for analysis.

\section{Multiparametric Data Analysis}

The recorded spike trains were analyzed for every concentration to obtain concentration-response effects of the compounds on multiple functional parameters. We report these effects with selected electrophysiological parameters.

The action potentials, or "spikes," were recorded in spike trains and are clustered in the so-called bursts. Bursts were quantitatively described via direct spike train analysis using the program NPWaveX (NeuroProof, Rostock, Germany). Bursts were defined with maximum spike intervals of $40 \mathrm{~ms}$ defining the start of a burst and maximum intervals to end a burst adjusted from 50 to $200 \mathrm{~ms}$.

For each 30 min of stable activity phase 30 min after substance application, we calculated 204 spike train parameters. Values were normalized to the respective baseline activity. Multiparametric results are represented in 4 categories (Table 1): general activity (mean spike rate, mean burst rate, and spike contrast), burst structure (mean spike number, burst area, and burst peak frequency), oscillation (CVtime of spike rate, CVtime of burst rate, and CVtime of burst peak frequency), and synchronicity (CVnet of spike rate, synchronization distances, and spike simplex mean).

\section{Statistical Analysis}

We produced cell suspensions from 3 independent animal preparations. Each suspension was plated to all wells of its own
Wohlrab/Stintzing/Schultz/Jügelt/ Schroeder 
Table 1. Descriptions of neurophysiological parameters shown in this study

\begin{tabular}{|c|c|c|}
\hline \multirow[t]{2}{*}{ General activity } & Spike rate & Number of spike events (action potentials) per second \\
\hline & Burst rate & Number of bursts (high-frequency clusters of spikes) per second \\
\hline \multirow[t]{2}{*}{ Burst structure } & Burst spike number & Number of spikes per burst \\
\hline & Burst area & Numerically integrated area \\
\hline Oscillatory behavior & SD values & $\begin{array}{l}\text { Standard deviation, that is, variability over time within the experimental episode (the lower the SDs, the } \\
\text { higher the regularity of bursting events) }\end{array}$ \\
\hline \multirow[t]{2}{*}{ Synchronicity } & CVnet & $\begin{array}{l}\text { Coefficient of variation or relative variability across the network; a lower CVnet reflects more synchronicity, } \\
\text { and a higher CVnet reflects less synchronicity }\end{array}$ \\
\hline & $\begin{array}{l}\text { Synchronization } \\
\text { distances }\end{array}$ & $\begin{array}{l}\text { Is defined as average distance of bursts within a population burst from population burst center. It is a } \\
\text { measure for the strength of synchronicity of a network in an inversely proportional manner }\end{array}$ \\
\hline
\end{tabular}

Table 2. Mean values for all compounds and parameters

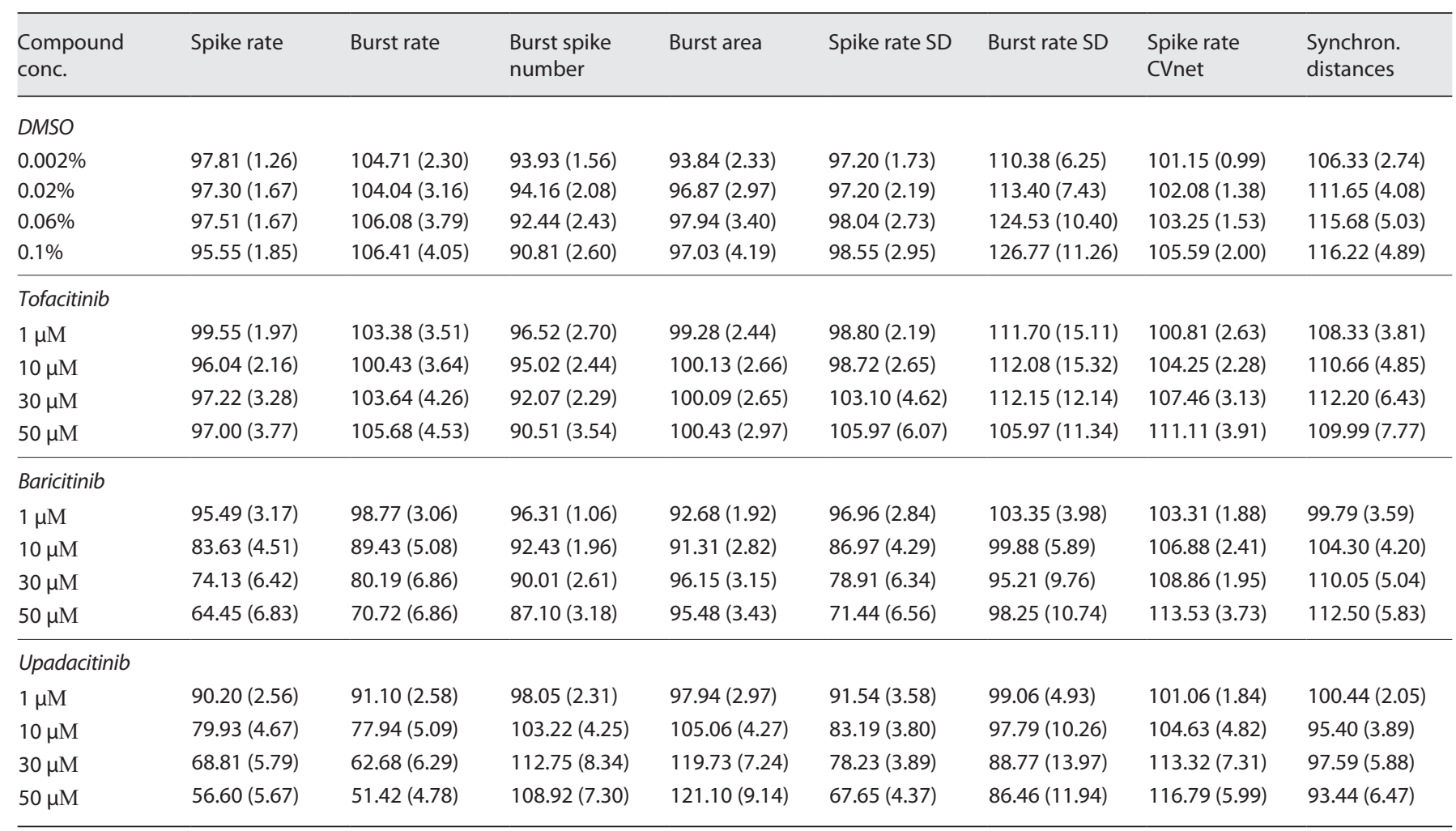

Values are in \% with SEM in parentheses. DMSO, dimethyl sulfoxide; SEM, standard error of mean.

MEA plate, creating individual cultures. Compound assignments were distributed across all preparations. Each culture was used once for a concentration-response experiment. The data were combined over all MEAs per test group with the following numbers of experiments: tofacitinib: 10 , baricitinib: 10 , upadacitinib: 10, and DMSO: 27.

JAKi and Itch
Concentration-response effects are shown as mean values \pm standard error of mean. The statistical significance of the effects of the compound versus vehicle-induced effects was assessed by oneway analysis of variance followed by Dunnett's post hoc adjustment. Direct compound comparisons used Student's unpaired $t$ test. $p<0.05$ was considered statistically significant. 


\section{DMSO $\square$ Tofacitinib $\square$ Baricitinib $\square$ Upadacitinib}

Spike rate

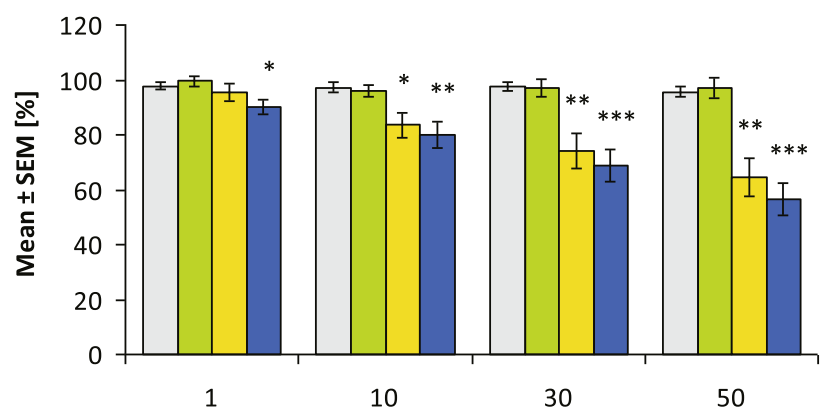

Burst spike number

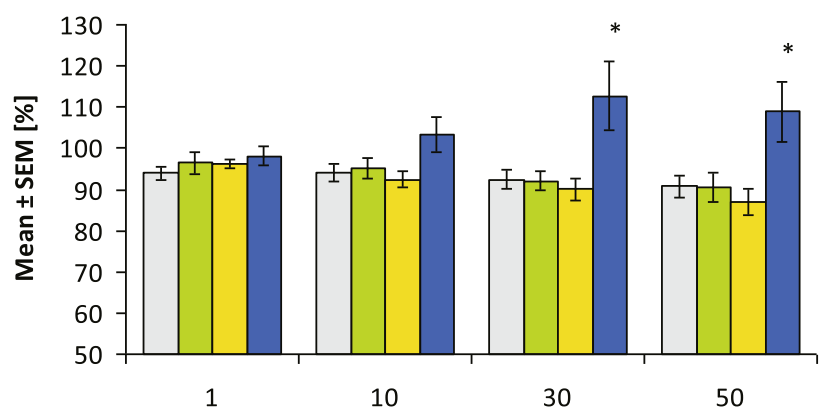

Spike rate SD

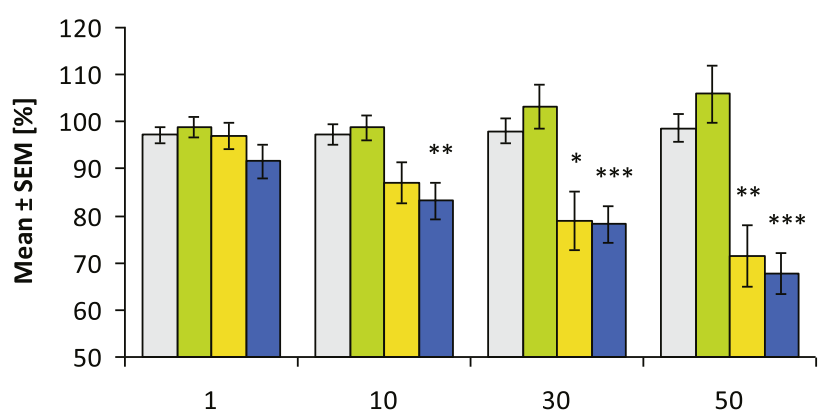

Spike rate CVnet

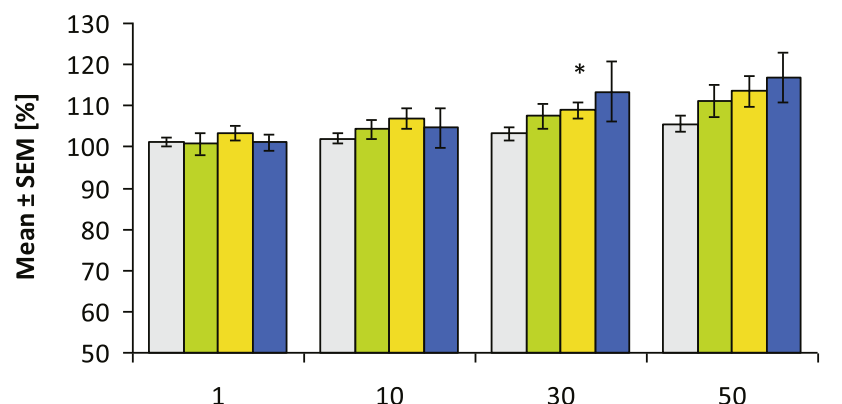

Concentration $[\mu \mathrm{M}]$
Burst rate

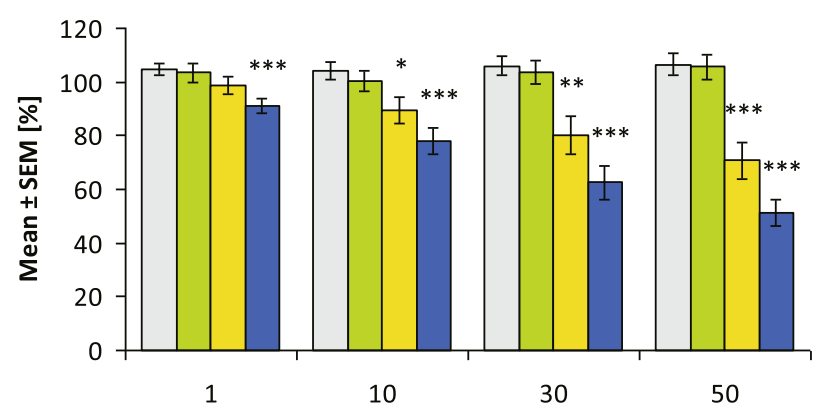

Burst area

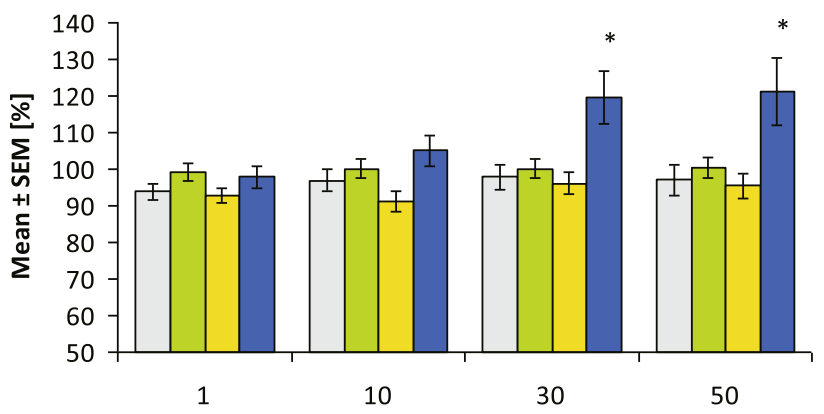

Burst rate SD

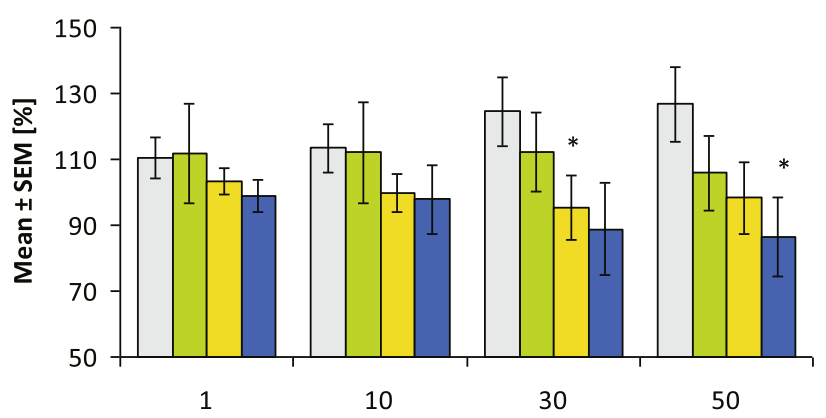

Synchronisation distances

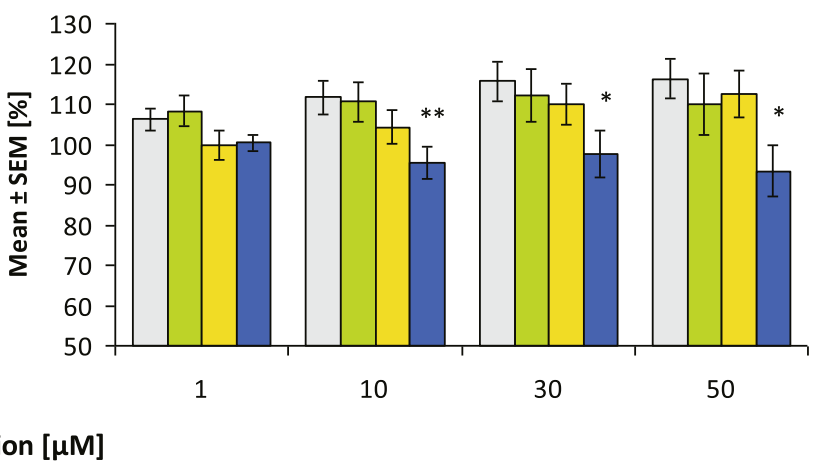

(For legend see next page.) 


\section{Results}

The results make it clear that JAKi can have a significant direct effect on the function of the neurosensory system. The quantity of influence depends on the receptor specificity of the substance used and its bioavailable dose.

The measurements are summarized in Table 2. Tofacitinib and the solvent DMSO did not have significant effects on the electrophysiological activity. Baricitinib had no effects at $1 \mu \mathrm{mol} / \mathrm{L}$ and caused a dose-dependent decrease at 10,30 , and $50 \mu \mathrm{mol} / \mathrm{L}$ to $99,89,80$, and $71 \%$ of the baseline burst rate (Fig. 2). Upadacitinib significantly reduced the burst rate in a dose-dependent manner at 1, 10, 30, and 50 $\mu \mathrm{mol} / \mathrm{L}$ to $91,78,63$, and $51 \%$ of the baseline. The spike rate was similarly affected by both compounds, with effects of upadacitinib being significantly stronger $(90,80,69$, and $57 \%)$ than baricitinib $(95,84,74$, and $64 \%)$ and already starting at $1 \mu \mathrm{mol} / \mathrm{L}$. Upadacitinib changed the burst structures while baricitinib did not. Bursts became longer (at 50 $\mu \mathrm{mol} / \mathrm{L}$, burst spike number increased significantly to $109 \%$, and burst area increased significantly to $121 \%)$. The burst peak frequency was unaffected (not shown), suggesting no influence on the spiking dynamics within bursts. The oscillatory behavior of the spiking and bursting was significantly more regular for upadacitinib and baricitinib (spike rate SD reduced at $50 \mu \mathrm{mol} / \mathrm{L}: 68 \%$ and $71 \%$ ). The regularity of the burst structure (burst peak frequency SD) was unaffected (not shown). The general activity levels remained homogenous within the networks with only numerical increases in spike rate $\mathrm{CVnet}$, the variability of the activity across neurons. Upadacitinib led to an increased synchronization between burst with the synchronization distance significantly reduced to $93 \%$. Baricitinib did not change this parameter.

\section{Discussion}

The antipruritic effect of JAKi, which has already been preclinically proven in animal models as well as in humans, especially in atopic dermatitis, is on the one hand

Fig. 2. Concentration-responses of DMSO (0.002-0.1\%), tofacitinib, baricitinib, and upadacitinib $(1-50 \mu \mathrm{M})$ on frontal cortex networks. Values are mean \pm SEM with statistical significance $(* / * * / * *$ for $p \leq 0.05 / 0.01 / 0.001)$. Values of rows $2-4$ are scaled to a minimum of $50 \%$. DMSO, dimethyl sulfoxide; SEM, standard error of mean.

JAKi and Itch mediated by a reduction of JAK-dependent cytokines involved in sensory perception (e.g., IL-31, IL-4, and IL-13) and on the other hand by the inhibition of the transient receptor potential cation channel subfamily $\mathrm{V}$ member 1 [13]. However, such effects have been demonstrated for both tofacitinib (pan-JAKi) and oclacitinib (JAK1i) and therefore do not appear to be specific to the JAK isotype. These data thus explain the antipruritic effects of JAKi in general, but do not indicate isotype selectivity or provide a basis for possible prioritization of JAKi or dose estimates for clinical development [19, 25].

The results of the present investigations now make it clear for the first time that in the comparison of the JAKi studied, the influence on electrophysical neuronal activity increases with isotype selectivity in relation to JAK1. This is also easy to understand due to the molecular receptor physiology known so far. The JAKi examined here differ significantly in the JAK-isoform selectivity, in the protein binding behavior, and in the regulation of cellular uptake mechanisms [23,26-28]. This indicates that especially the receptor types activated by type 2 cytokines are involved in the neurosensory processes [29]. Whether the observation of JAK1-associated efficacy also coincides with the clinical parameters for the reduction of itching or with the inhibition of inflammatory cascades, however, cannot be assessed at present. Therefore, it is of great interest to evaluate the growing amount of data on clinical outcome parameters in different indications.

In addition, it is also possible to derive information for the evaluation of data on cutaneous bioavailability of JAKi [30]. The comparison of dose-dependent pharmacokinetic profiles after systemic or topical application would be of great interest here [31]. Regarding atopic dermatitis, for example, the focus on systemic bioavailability for the therapy of inflammatory cascades and on cutaneous bioavailability for antipruritic effects could possibly be appropriate. Moreover, it seems questionable whether the dose levels for an optimized anti-inflammatory effect of the individual JAKi are identical to those of an antipruritic effect. In addition, the variability of bioavailability depending on the indication, population, and age should be considered [32-34]. Due to the large number of JAKi in development for different indications and the inhomogeneous data situation, only head-to-head studies will be able to prove relevant differences in clinically effective preparations. However, the significance of the data presented is limited by methodological constraints. The studies were performed on cells from mice, and it is unclear to what extent the results can be extrapolated to humans. Furthermore, cortex cells without peripheral neu- 
rons were used as a model, so that it remains unclear whether the effects can also be demonstrated on the entire neurosensory signaling cascade.

\section{Statement of Ethics}

Experiments were carried out in accordance with the EU Directive 2010/63/EU on the protection of animals used for scientific purposes (Certification File No. 7221.3-2).

\section{Conflict of Interest Statement}

J.W. has received fees for lecturing and/or consulting and/or received funding for scientific projects and/or clinical studies from Abbvie, Leo, Lilly, Novartis, and Pfizer. D.S. declares no conflicts of interest. L.S., K.J., and O.S. are employees of NeuroProof GmbH.

\section{Funding Sources}

The study was partially sponsored by NeuroProof GmbH, Rostock, Germany.

\section{Author Contributions}

J.W. provided oversight for the project, defined the scope, directed the work, and wrote together with D.S. the manuscript. L.S., K.J., and O.S. performed experiments and data analysis and wrote the methodical part of the manuscript.

\section{Data Availability Statement}

The authors confirm that the data supporting the findings of this study are available within the article.

\section{References}

1 Yosipovitch G, Misery L, Proksch E, Metz M, Ständer S, Schmelz M. Skin barrier damage and itch: review of mechanisms, topical management and future directions. Acta Derm Venereol. 2019;99:1201-9.

2 Misery L, Weisshaar E, Brenaut E, Evers AWM, Huet F, Stander S, et al. Special interest group on sensitive skin of the international forum for the study of I: pathophysiology and management of sensitive skin: position paper from the special interest group on sensitive skin of the international forum for the study of itch (ifsi). J Eur Acad Dermatol Venereol. 2020;34:222-9.

3 Cevikbas F, Lerner EA. Physiology and pathophysiology of itch. Physiol Rev. 2020; 100:945-82.

4 Abdo H, Calvo-Enrique L, Lopez JM, Song J, Zhang MD, Usoskin D, et al. Specialized cutaneous schwann cells initiate pain sensation. Science. 2019;365:695-9.

5 Oetjen LK, Mack MR, Feng J, Whelan TM, Niu H, Guo CJ, et al. Sensory neurons co-opt classical immune signaling pathways to mediate chronic itch. Cell. 2017;171:217-e13.

6 O'Shea JJ, Schwartz DM, Villarino AV, Gadina M, McInnes IB, Laurence A. The jakstat pathway: impact on human disease and therapeutic intervention. Annu Rev Med. 2015;66:311-28.

7 Suter MR, Wen YR, Decosterd I, Ji RR. Do glial cells control pain? Neuron Glia Biol. 2007;3:255-68.

8 Peteri UK, Niukkanen M, Castrén ML. Astrocytes in neuropathologies affecting the frontal cortex. Front Cell Neurosci. 2019;13: 44.

9 Molet J, Mauborgne A, Diallo M, Armand V, Geny D, Villanueva L, et al. Microglial janus kinase/signal transduction and activa- tor of transcription 3 pathway activity directly impacts astrocyte and spinal neuron characteristics. J Neurochem. 2016;136: 133-47.

10 Vogelaar CF, Hoekman MF, Gispen WH, Burbach JP. Homeobox gene expression in adult dorsal root ganglia during sciatic nerve regeneration: is regeneration a recapitulation of development? Eur J Pharmacol. 2003;480:233-50.

11 Grace PM, Hutchinson MR, Maier SF, Watkins LR. Pathological pain and the neuroimmune interfaceace. Nat Rev Immunol. 2014 Apr;14(4):217-31.

12 Fowler E, Yosipovitch G. A new generation of treatments for itch. Acta Derm Venereol. 2020;100:adv00027.

13 Fukuyama T, Ganchingco JR, Mishra SK, Olivry T, Rzagalinski I, Volmer DA, et al. Janus kinase inhibitors display broad antiitch properties: a possible link through the trpv1 receptor. J Allergy Clin Immunol. 2017;140:306-e3.

14 Hanisch UK, Lyons SA, Prinz M, Nolte C, Weber JR, Kettenmann H, et al. Mouse brain microglia express interleukin-15 and its multimeric receptor complex functionally coupled to janus kinase activity. J Biol Chem. 1997;272:28853-60.

$15 \mathrm{Du}$ L, Hu X, Yang W, Yasheng H, Liu S, Zhang W, et al. Spinal il-33/st2 signaling mediates chronic itch in mice through the astrocytic jak2-stat3 cascade. Glia. 2019;67: 1680-93.

16 Fukuyama T, Tschernig T, Qi Y, Volmer DA, Bäumer W. Aggression behaviour induced by oral administration of the januskinase inhibitor tofacitinib, but not oclacitinib, under stressful conditions. Eur J Pharmacol. 2015;764:278-82.
17 Durmus S, Xu N, Sparidans RW, Wagenaar E, Beijnen JH, Schinkel AH. P-glycoprotein (mdr1/abcb1) and breast cancer resistance protein (bcrp/abcg2) restrict brain accumulation of the jak1/2 inhibitor, cyt387. Pharmacol Res. 2013;76:9-16. .

18 Barar J, Rafi MA, Pourseif MM, Omidi Y. Blood-brain barrier transport machineries and targeted therapy of brain diseases. Bioimpacts. 2016;6:225-48.

19 Arora CJ, Khattak FA, Yousafzai MT, Ibitoye BM, Shumack S. The effectiveness of jak inhibitors in treating atopic dermatitis: a systematic review and meta-analysis. Dermatol Ther. 2020 Jul;33(4):e13685.

20 Stander S, Luger T, Cappelleri JC, Bushmakin AG, Mamolo C, Zielinski MA, et al. Validation of the itch severity item as a measurement tool for pruritus in patients with psoriasis: results from a phase 3 tofacitinib program. Acta Derm Venereol. 2018;98:340-5.

21 Vaa BE, Tefferi A, Gangat N, Pardanani A, Lasho TL, Finke CM, et al. Pruritus in primary myelofibrosis: management options in the era of jak inhibitors. Ann Hematol. 2016;95:1185-9.

22 Xu L, Feng J, Gao G, Tang H. Momelotinib for the treatment of myelofibrosis. Expert Opin Pharmacother. 2019;20:1943-51.

23 Choy EH. Clinical significance of janus kinase inhibitor selectivity. Rheumatology. 2019;58:1122-62.

24 Parenti C, Turnaturi R, Aricò G, GramowskiVoss A, Schroeder OH, Marrazzo A, et al. The multitarget opioid ligand lp1's effects in persistent pain and in primary cell neuronal cultures. Neuropharmacology. 2013;71:70-82.

25 Fowler E, Yosipovitch G. Chronic itch management: therapies beyond those targeting the immune system. Ann Allergy Asthma Immunol. 2019;123:158-65. 
26 Abdelhameed AS, Alam P, Khan RH. Binding of janus kinase inhibitor tofacitinib with human serum albumin: mulri-technique approach. J Biomol Struct Dyn. 2016;34:203744.

27 Amrhein J, Drynda S, Schlatt L, Karst U, Lohmann CH, Ciarimboli G, et al. Tofacitinib and baricitinib are taken up by different uptake mechanisms determining the efficacy of both drugs in RA. Int J Mol Sci. 2020 Sep 10;21(18):6632.

28 McInnes IB, Byers NL, Higgs RE, Lee J, Macias WL, Na S, et al. Comparison of baricitinib, upadacitinib, and tofacitinib mediated regulation of cytokine signaling in human leukocyte subpopulations. Arthritis Res Ther. 2019;21(183):183-10.
29 Wynn TA. Type 2 cytokines: mechanisms and therapeutic strategies. Nat Rev Immunol. 2015;15:271-82.

30 Veeravalli V, Dash RP, Thomas JA, Babu RJ, Madgula LMV, Srinivas NR. Critical assessment of pharmacokinetic drug-drug interaction potential of tofacitinib, baricitinib and upadacitinib, the three approved janus kinase inhibitors for rheumatoid arthritis treatment. Drug Saf. 2020 Aug;43(8):71125.

31 Purohit VS, Ports WC, Wang C, Riley S. Systemic tofacitinib concentrations in adult patients with atopic dermatitis treated with $2 \%$ tofacitinib ointment and application to pediatric study planning. J Clin Pharmacol. 2019;59(6):811-20.
32 Nader A, Stodtmann S, Friedel A, Mohamed MF, Othman AA. Pharmacokinetics of upadacitinib in healthy subjects and subjects with rheumatoid arthritis, crohn's disease, ulcerative colitis, or atopic dermatitis: population analyses of phase 1 and 2 clinical trials. J Clin Pharmacol. 2020;60:528-39.

33 Miyoshi S, Krishnaswami S, Toyoizumi S, Nakamura H, Zwillich SH. Phase 1 dose-escalation study to evaluate the pharmacokinetics, safety, and tolerability of tofacitinib in japanese healthy volunteers. Clin Pharmacol Drug Dev. 2020;9:11-20. .

34 Kim H, Brooks KM, Tang CC, Wakim P, Blake M, Brooks SR, et al. Pharmacokinetics, pharmacodynamics, and proposed dosing of the oral jak1 and jak2 inhibitor baricitinib in pediatric and young adult candle and savi patients. Clin Pharmacol Ther. 2018;104:364-73. 